\title{
Refractory Uterine Corpus Carcinoma
}

National Cancer Institute

\section{Source}

National Cancer Institute. Refractory Uterine Corpus Carcinoma. NCI Thesaurus. Code C150092.

Uterine corpus carcinoma that is resistant to treatment. 